\title{
Prediction of Epitopes in the Proteome of Helicobacter pylori
}

\author{
Elkin Navarro-Quiroz ${ }^{1,2}$, Roberto Navarro-Quiroz ${ }^{3}$, Pierine España-Puccini ${ }^{2,4}$, José Luis Villarreal ${ }^{4,5}$, Anderson \\ Díaz Perez ${ }^{1,9}$, Cecilia Fernandez Ponce ${ }^{6}$, Jorge Bilbao ${ }^{5}$, Lucy Vasquez ${ }^{7} \&$ Dary Luz Mendoza ${ }^{8}$ \\ ${ }^{1}$ Universidad Simón Bolívar, Barranquilla, Colombia \\ ${ }^{2}$ E Biotechnology, Barranquilla, Colombia \\ ${ }^{3}$ Centro de Investigación en Salud para el Trópico, Universidad Cooperativa de Colombia, Santa Marta, Colombia \\ ${ }^{4}$ Universidad del Norte, Barranquilla, Colombia \\ ${ }^{5}$ Universidad Libre, sede Barranquilla, Colombia \\ ${ }^{6}$ Universidad de Cádiz, Cádiz, España \\ ${ }^{7}$ Clínica la misericordia, Barranquilla, Colombia \\ ${ }^{8}$ Universidad del Atlántico, Barranquilla, Colombia \\ ${ }^{9}$ Universidad Rafael Nuñez, Cartagena, Colombia \\ Correspondence: Elkin Navarro-Quiroz, Profesor asistente, Universidad Simón Bolívar, Carrera 59 No. 59-65, \\ Colombia.Tel: 300-532-4251. E-mail: enavarro26@unisimonbolivar.edu.co
}

Received: May 12, 2018 Accepted: June 3, 2018 Online Published: June 11, 2018

doi:10.5539/gjhs.v10n7p148 URL: https://doi.org/10.5539/gjhs.v10n7p148

\begin{abstract}
Helicobacter pylori (H. pylori) is classified by the World Health Organization (WHO) as a group I carcinogen and is one of the most efficient human pathogens with over half of the world's population colonized by this gram-negative spiral bacterium. H. pylori can cause a chronic infection in the stomach during early childhood that persists throughout life due to diverse mechanisms of immune response evasion. H. pylori has several factors strongly associated with increased risk of disease such as toxins, adhesins, and chemoattractants, some of which are highly polymorphic, phase variable, and have different functions. Conventional treatments involve the use of antibiotics. However, treatment frequently fails due to the resistance H. pylori has progressively developed to antibiotics. This creates the need for different treatments made possible by identifying new therapeutic targets in the pathogen's genome.

The purpose of this study was an in silico prediction of T- and B- epitopes in H. pylori proteins. Twenty-two external membrane proteins from H. pylori Strain 26695 (accession number NC_000915) were identified using the web tool Vaxign (http://www.violinet.org/vaxign/). A total of one-hundred epitopes (60 class I epitopes and 40 class II epitopes) that could be used to develop novel non-antibiotics drugs for an $\mathrm{H}$. pylori infection were predicted.
\end{abstract}

Keywords: Helicobacter pylori, epitopes, chronic infection in the stomach

\section{Introducción}

Helicobacter pylori ( H. pylori ) was first isolated by Warren and Marshall in 1982 (Ford \& Axon, 2010), It is a slow-growing, Gram-negative, flagellated spiral-shaped microaerophilic organism (Jemilohun \& Otegbayo, 2016).It has two to six flagella that provide mobility to withstand the rhythmic contractions in the stomach so they are able to penetrate the gastric mucosa (Spohn \& Scarlato, 2001). It measures around $2.4-4.0 \mu \mathrm{m}$ long and 0.5-1.0 $\mu \mathrm{m}$ wide (Refaeli et al., 2018).

H. pylori is an important pathogen in Public Health worldwide as one of the main causes of gastric diseases(Hooi et al., 2017). Several studies have shown that $H$. pylori is present in about $50 \%$ of the world population (Pilotto \& Franceschi, 2014). In developing countries, the prevalence of infection can exceed $90 \%$ in adulthood(Hooi et al., 2017).

The host immune response induced by $H$. pylori is characterized by neutrophil recruitment followed by macrophages, mast cells, eosinophils, and T and B lymphocytes (Ayraud, Janvier, \& Fauchère, 2002). Additionally, 
there is an increase of IL-1, IL-8, and IL-6, and a reduction in the production of IL-17 (Kabir, 2011).

The current treatment of an $H$. pylori infection uses a combination of at least three compounds which include a proton pump inhibitor and two antibiotics. This treatment's decline in efficiency has been documented mainly due to its increased resistance to antibiotics (Fallone et al., 2016; Mégraud, 2012). Therefore, it is imperative to develop new therapeutic alternatives.

The rational design of a vaccine based on In silico prediction epitopes is a promising way to approach this problem (Moss et al., 2011). It has been demonstrated that surface proteins of some microorganisms have immunogenic epitopes, which could be used to design and develop novel, safe and efficient vaccines against pathogens (Ni, Wang, Liu, \& $\mathrm{Lu}, 2010$ ). However, the greatest requirement for the candidate epitopes is their ability to bind to molecules of Major Histocompatibility Complex (MHC) class I and / or class II. These molecules cause the epitopes to come to the cell surface to be subsequently recognition by the lymphocytes (Liao et al., 2009).

Vaxign is an online design system that predicts target proteins for vaccine design based on genome sequences using reverse vaccinology strategy (He, Xiang, \& Mobley, 2010). The preestablished characteristics in Vaxign include the subcellular localization of the white protein and identifying epitopes that bind to MHC class I and class II (He et al., 2010; Xiang \& He, 2009).

This study aimed to predict T- and B- epitopes from outer membrane proteins of Helicobacter pylori that can be used in later work to develop a vaccine against this microorganism or for alternative therapies such as passive immunization.

\section{Methodology}

\subsection{Obtaining Genomic Sequences of H. pylori}

The complete genome sequence of H. pylori was researched in RefSeq (Pruitt, Tatusova, \& Maglott, 2005) and GenBank (Benson, Karsch-Mizrachi, Lipman, Ostell, \& Wheeler, 2006) databases..

\subsection{Identification of Conserved Sequences}

The OrthoMCL algorithm was used to find conserved proteins (Yu et al., 2010) and SPAAN software (Sachdeva, Kumar, Jain, \& Ramachandran, 2005). The transmembrane helix topology analysis was carried out using optimized HMMTOP (Xiang \& He, 2009) is freely available to non-commercial users at http://www.enzim.hu/hmmtop. Source code is also available upon request to academic users.

\subsection{Identification of Conserved Sequences}

The OrthoMCL algorithm was used to find conserved proteins (Chen, Mackey, Stoeckert, \& Roos, 2006), OrthoMCL provides a scalable method for constructing orthologous groups across multiple eukaryotic taxa, using a Markov Cluster algorithm to group (putative) orthologs and paralogs. This method performs similarly to the INPARANOID algorithm when applied to two genomes, but can be extended to cluster orthologs from multiple species. A cut off of E-105 was used as the default value for all proteins that could cross-react with human but mouse and/or pig proteins were discarded.

\subsection{Prediction of Epitopes}

For H. pylori protein, the Vaxitop pipeline was used for the prediction of epitopes (http://www.violinet.org/vaxign/vaxitop), following the methodology described above by (Xiang \& He, 2013). Calculations were done using a cut-off point of $\mathrm{p} \leq 0.05$, which provides high sensibility, specificity and balance (He et al., 2010).

\section{Results}

\subsection{H. pylori Genomes}

A total of $683 \mathrm{H}$. pylori genomes were found, of which only 61 sequences corresponded to genomes strains of this microorganism, with an average of 1,571 and 1,454 protein genes (Table 1). 
Table 1. Genomes of H. pylori strains

\begin{tabular}{|c|c|c|c|}
\hline Strain & Assembly & Genes & Proteínas \\
\hline Helicobacter pylori 26695 & GCA_000008525.1 & 1555 & 1445 \\
\hline Helicobacter pylori J99 & GCA_000008785.1 & 1607 & 1469 \\
\hline Helicobacter pylori Shi470 & GCA_000020245.1 & 1584 & 1442 \\
\hline Helicobacter pylori B38 & GCA_000091345.1 & 1545 & 1432 \\
\hline Helicobacter pylori 51 & GCA_000011725.1 & 1545 & 1433 \\
\hline Helicobacter pylori 52 & GCA_000023805.1 & 1522 & 1392 \\
\hline Helicobacter pylori 908 & GCA_000148665.1 & 1501 & 1360 \\
\hline Helicobacter pylori SJM180 & GCA_000148855.1 & 1579 & 1488 \\
\hline Helicobacter pylori Cuz20 & GCA_000148895.1 & 1580 & 1487 \\
\hline Helicobacter pylori $35^{a}$ & GCA_000178935.2 & 1530 & 1414 \\
\hline Helicobacter pylori India 7 & GCA_000185185.1 & 1602 & 1487 \\
\hline Helicobacter pylori F16 & GCA_000270005.1 & 1531 & 1421 \\
\hline Helicobacter pylori F57 & GCA_000270065.1 & 1560 & 1456 \\
\hline Helicobacter pylori 2017 & GCA_000192315.1 & 1506 & 1382 \\
\hline Helicobacter pylori 2018 & GCA_000192335.1 & 1516 & 1393 \\
\hline Helicobacter pylori 83 & GCA_000213135.1 & 1562 & 1441 \\
\hline Helicobacter pylori Puno135 & GCA_000224555.1 & 1585 & 1493 \\
\hline Helicobacter pylori Shi417 & GCA_000277365.1 & 1586 & 1490 \\
\hline Helicobacter pylori Shil 69 & GCA_000277385.1 & 1559 & 1460 \\
\hline Helicobacter pylori Shil12 & GCA_000277405.1 & 1607 & 1499 \\
\hline Helicobacter pylori PeCan 18 & GCA_000277425.1 & 1581 & 1480 \\
\hline Helicobacter pylori 26695 & GCA_000307795.1 & 1632 & 1538 \\
\hline Helicobacter pylori Rif1 & GCA_000307815.1 & 1630 & 1532 \\
\hline Helicobacter pylori Rif2 & GCA_000307835.1 & 1630 & 1534 \\
\hline Helicobacter pylori OK113 & GCA_000348865.1 & 1553 & 1418 \\
\hline Helicobacter pylori UM032 & GCA_000392455.3 & 1549 & 1438 \\
\hline Helicobacter pylori UM299 & GCA_000392475.3 & 1550 & 1439 \\
\hline Helicobacter pylori UM037 & GCA_000392515.3 & 1634 & 1509 \\
\hline Helicobacter pylori UM066 & GCA_000392535.3 & 1587 & 1474 \\
\hline Helicobacter pylori UM298 & GCA_000439295.2 & 1551 & 1440 \\
\hline Helicobacter pylori SouthAfrica20 & GCA_000590775.1 & 1552 & 1361 \\
\hline Helicobacter pylori $26695-1$ & GCA_000829095.1 & 1629 & 1539 \\
\hline Helicobacter pylori 26695-1CL & GCA_000829115.1 & 1628 & 1538 \\
\hline Helicobacter pylori $26695-1 \mathrm{CH}$ & GCA_000829135.1 & 1628 & 1538 \\
\hline Helicobacter pylori BM012A & GCA_000498315.1 & 1632 & 1495 \\
\hline Helicobacter pylori BM012S & GCA_000498335.1 & 1632 & 1495 \\
\hline Helicobacter pylori oki102 & GCA_000600045.1 & 1566 & 1464 \\
\hline Helicobacter pylori oki112 & GCA_000600085.1 & 1569 & 1456 \\
\hline Helicobacter pylori oki 128 & GCA_000600125.1 & 1496 & 1364 \\
\hline Helicobacter pylori oki154 & GCA_000600145.1 & 1543 & 1412 \\
\hline
\end{tabular}




\begin{tabular}{llll}
\hline Strain & Assembly & Genes & Proténas \\
\hline Helicobacter pylori oki422 & GCA_000600165.1 & 1570 & 1441 \\
Helicobacter pylori oki673 & GCA_000600185.1 & 1534 & 1400 \\
Helicobacter pylori oki828 & GCA_000600205.1 & 1536 & 1399 \\
Helicobacter pylori oki898 & GCA_000600225.1 & 1563 & 1463 \\
Helicobacter pylori J166 & GCA_000685625.1 & 1569 & 1466 \\
Helicobacter pylori & GCA_000685665.1 & 1551 & 1453 \\
Helicobacter pylori & GCA_000685705.1 & 1626 & 1490 \\
Helicobacter pylori & GCA_000685745.1 & 1551 & 1452 \\
Helicobacter pylori NY40 & GCA_000828955.1 & 1643 & 1476 \\
Helicobacter pylori & GCA_000817025.1 & 1541 & 1413 \\
Helicobacter pylori $26695-1$ & GCA_000826985.1 & 1630 & 1542 \\
Helicobacter pylori & GCA_000827025.1 & 1631 & 1542 \\
Helicobacter pylori J99 & GCA_000982695.1 & 1594 & 1491 \\
Helicobacter pylori & GCA_001433495.1 & 1617 & 1451 \\
Helicobacter pylori & GCA_001653375.1 & 1584 & 1456 \\
Helicobacter pylori & GCA_001653395.1 & 1573 & 1441 \\
Helicobacter pylori & GCA_001653415.1 & 1590 & 1471 \\
Helicobacter pylori & GCA_001653435.1 & 1462 & 1353 \\
Helicobacter pylori & GCA_001653455.1 & 1498 & 1392 \\
Helicobacter pylori & GCA_001653475.1 & 1491 & 1354 \\
\hline & & & \\
\hline
\end{tabular}

\subsection{H. pylori Vaccine Candidate Protein Predition}

H. pylori strain 26695 was arbitrarily selected for the prediction of protein candidates. For this strain, a total of 1,445 proteins have been described. In this study, 42 outer membrane proteins were identified, with a sticky probability $\geq 0.51$. These proteins had no significant homology with pig, mouse or human proteins (Table 2 ). The outer membrane proteins with a greater likelihood adhesin (0.737) were: NP_207714.1 (a protein with fusion functions) and NP_207405.1 (a toxin-like protein).

Table 2. Selection of conserved outer membrane proteins of Helicobacter pylori, with probability of adherence $\geq 0.51$

\begin{tabular}{lll}
\hline Protein Accession & Localization & Adhesin Probability \\
\hline NP_206827.1 & Outer Membrane & 0.685 \\
NP_207052.1 & Outer Membrane & 0.656 \\
NP_207115.1 & Outer Membrane & 0.705 \\
NP_207284.1 & Outer Membrane & 0.681 \\
NP_207405.1 & Outer Membrane & 0.737 \\
NP_207432.1 & Outer Membrane & 0.544 \\
NP_207480.1 & Outer Membrane & 0.620 \\
NP_207504.1 & Outer Membrane & 0.610 \\
NP_207516.1 & Outer Membrane & 0.653 \\
NP_207519.1 & Outer Membrane & 0.640 \\
NP_207575.1 & Outer Membrane & 0.517 \\
\hline
\end{tabular}




\begin{tabular}{lll}
\hline NP_207581.1 & Outer Membrane & 0.578 \\
NP_207600.1 & Outer Membrane & 0.662 \\
NP_207670.1 & Outer Membrane & 0.555 \\
NP_207689.1 & Outer Membrane & 0.662 \\
NP_207704.1 & Outer Membrane & 0.669 \\
NP_207705.1 & Outer Membrane & 0.684 \\
NP_207706.1 & Outer Membrane & 0.629 \\
NP_207714.1 & Outer Membrane & 0.737 \\
NP_208191.1 & Outer Membrane & 0.687 \\
NP_208244.1 & Outer Membrane & 0.636 \\
NP_208303.1 & Outer Membrane & 0.628 \\
\hline
\end{tabular}

The outer membrane proteins with a greater likelihood adhesin (0.737) were: NP_207714.1 (a protein with fusion functions) and NP_207405.1 (a toxin-like protein).

Epitope Prediction of MHC Class I and II

Vaxitop (Xiang \& He, 2013) was used to predict MHC class I and II binding epitopes. Likewise, other programs used to identify epitopes are listed in supplementary Table 1.

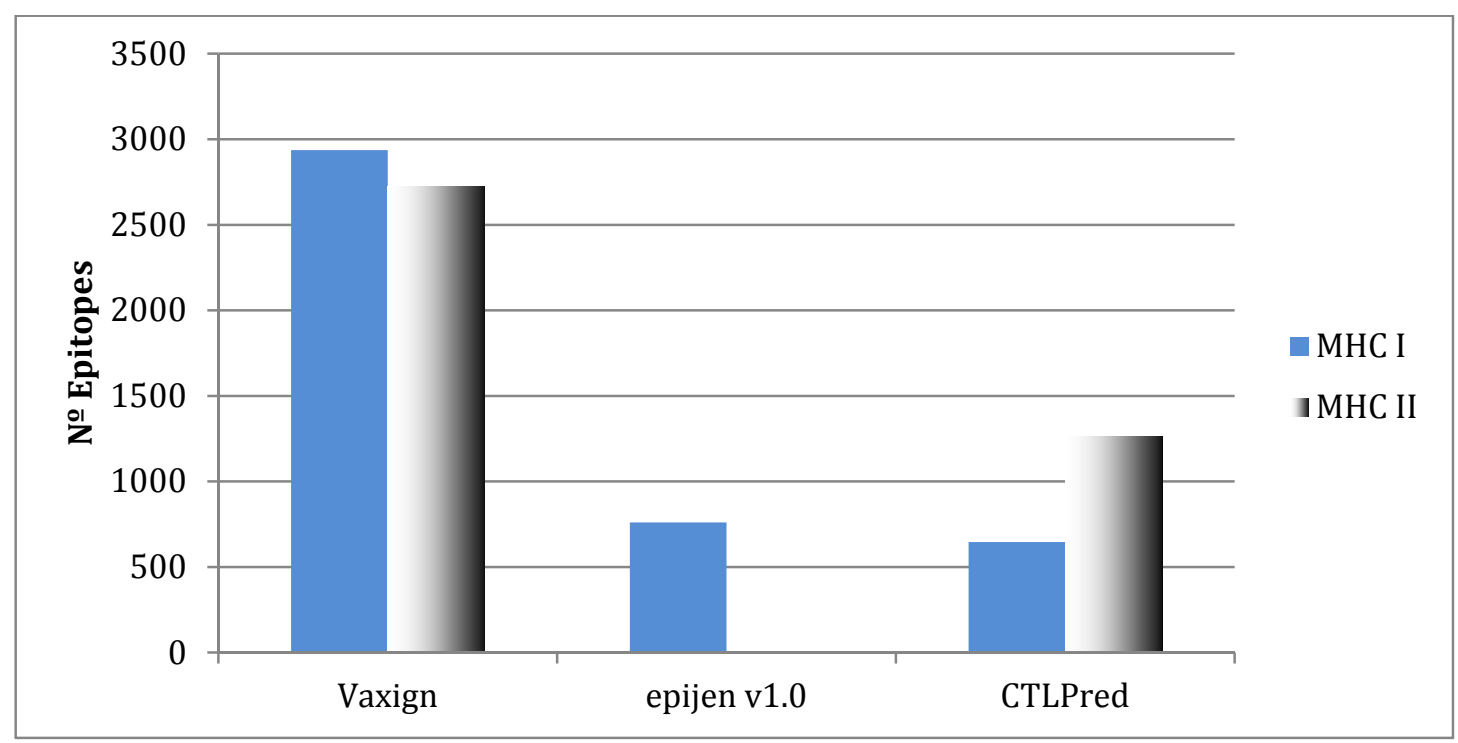

Figure 1. Total number of epitopes MHC I and MHC II predicted with different software

The greatest number of epitopes was obtained with Vaxign software (PSSM) with 2881 class I epitopes and 2634 class II epitopes. (Figure 1) The VacA protein (WP_000874574.1), an H. pylory outer membrane protein toxin, presented the highest number of epitopes, as follows: 60 epitopes for MHC class I (Table 3), and 40 epitopes is for MHC class II (Table 4). 
Table 3. Predicted epitopes for MHC I in proteins of Helicobacter pylori.

\begin{tabular}{|c|c|c|c|c|c|}
\hline $\mathrm{N}^{0}$ de acceso proteína & Epítope & alelo MHC & aminoacido Inicial & aminoacido final & P-valor \\
\hline \multirow{3}{*}{ WP_000726314.1 } & TTLNEACPWL & HLA-A*01:01 & 264 & 273 & 0.0159 \\
\hline & GTSWLNSQYV & HLA-A*02:01 & 619 & 628 & 0.0244 \\
\hline & LYSVYLNYVF & HLA-A*24:02 & 700 & 709 & $6.50 \mathrm{E}-05$ \\
\hline \multirow{3}{*}{ WP_000713712.1 } & LYSVYLNYVF & HLA-A $* 24: 02$ & 734 & 743 & $6.50 \mathrm{E}-05$ \\
\hline & SWLNSEYVNL & HLA-A*24:02 & 655 & 664 & 0.00732 \\
\hline & DTLVNFKSRY & HLA-A*01:01 & 479 & 488 & 0.00163 \\
\hline \multirow{3}{*}{ WP_001115603 } & QYRGFSWKIL & HLA-A*24:02 & 384 & 393 & 0.00022 \\
\hline & KALMVADLKY & HLA-A*01:01 & 233 & 242 & 0.0173 \\
\hline & ITYDTNPNFN & HLA-A*01:01 & 267 & 276 & 0.0777 \\
\hline \multirow{3}{*}{ WP_000709741.1 } & RVKGLSIFYK & HLA-A*03:01 & 168 & 177 & 0.00044 \\
\hline & DYKRVVSVYL & HLA-A $* 24: 02$ & 286 & 295 & 0.0095 \\
\hline & LPYGFNTDLL & HLA-B*07:02 & 142 & 151 & 0.00792 \\
\hline \multirow{3}{*}{ WP_000822057.1 } & FIFDMMYTYK & HLA-A*03:01 & 645 & 654 & 0.00013 \\
\hline & PGLRYTFLNY & HLA-A*01:01 & 465 & 474 & 0.0247 \\
\hline & NVFGGVINVI & HLA-A*02:01 & 155 & 164 & 0.0184 \\
\hline \multirow{3}{*}{ WP_001228436.1 } & GYQNYFNDFI & HLA-A $* 24: 02$ & 508 & 517 & 0.0009 \\
\hline & QLTIENFLPY & HLA-A*01:01 & 95 & 104 & 0.0392 \\
\hline & FGDNLKTINL & HLA-A*01:01 & 333 & 342 & 0.0134 \\
\hline \multirow{3}{*}{ WP_001092132.1 } & KSDKAALGLY & HLA-A*01:01 & 79 & 88 & 0.00083 \\
\hline & YATGRFGNFY & HLA-A*01:01 & 332 & 341 & 0.018 \\
\hline & ATYRSNVANL & HLA-B*07:02 & 172 & 181 & 0.00582 \\
\hline \multirow{3}{*}{ WP_010875534.1 } & LYSVYLNYVF & HLA-A $* 24: 02$ & 597 & 606 & $6.50 \mathrm{E}-05$ \\
\hline & RLYSVYLNYV & HLA-A*02:01 & 596 & 605 & 0.0002 \\
\hline & ITSTGPVTDY & HLA-A*01:01 & 203 & 212 & 0.00057 \\
\hline \multirow{3}{*}{ WP_000911476.1 } & FYSYGDKFHL & HLA-A*24:02 & 150 & 159 & 0.00018 \\
\hline & YVLYNSYLFY & HLA-A*01:01 & 142 & 151 & 0.00031 \\
\hline & VYRGFLWGIL & HLA-A*24:02 & 372 & 381 & 0.00063 \\
\hline \multirow{3}{*}{ WP_001108275.1 } & YMMDANAFTV & HLA-A*02:01 & 401 & 410 & $2.67 \mathrm{E}-05$ \\
\hline & AYMQVDFTEL & HLA-A*24:02 & 67 & 76 & 0.00022 \\
\hline & FSADIKFEYY & HLA-A*01:01 & 452 & 461 & 0.00023 \\
\hline \multirow{3}{*}{ WP_010875548.1 } & FYFNYQRSYI & HLA-A*24:02 & 540 & 549 & 0.0002 \\
\hline & NTFKAYYQYY & HLA-A*01:01 & 275 & 284 & 0.00035 \\
\hline & QYNSYHPGTL & HLA-A $* 24: 02$ & 285 & 294 & 0.0016 \\
\hline \multirow{3}{*}{ WP_000479960.1 } & GLDYCGFDIY & HLA-A*01:01 & 677 & 686 & 0.00028 \\
\hline & DSLFEYGFNY & HLA-A*01:01 & 404 & 413 & 0.00613 \\
\hline & RPLRSNAIGL & HLA-B*07:02 & 257 & 266 & 0.0016 \\
\hline \multirow{3}{*}{ WP_000753173.1 } & FFDYNHAFIK & HLA-A*03:01 & 557 & 566 & 0.0174 \\
\hline & KQASIIITTL & HLA-A*02:01 & 260 & 269 & 0.0244 \\
\hline & QELGRNPFRK & HLA-A*03:01 & 509 & 518 & 0.0715 \\
\hline
\end{tabular}




\begin{tabular}{|c|c|c|c|c|c|}
\hline & FYTKIGYKQF & HLA-A*24:02 & 350 & 359 & 0.00069 \\
\hline \multirow[t]{3}{*}{ WP_000592437.1 } & LSTIGSQTNY & HLA-A*03:01 & 149 & 158 & 0.0477 \\
\hline & QTYSTQAIQY & HLA-A*03:01 & 206 & 215 & 0.0536 \\
\hline & VYLTYMNSFL & HLA-A $* 24: 02$ & 197 & 206 & 0.00038 \\
\hline \multirow[t]{3}{*}{ WP_000812546.1 } & NQLGNLIDLY & HLA-A*01:01 & 105 & 114 & 0.0159 \\
\hline & TYGVGTDVLY & HLA-A*01:01 & 410 & 419 & 0.0203 \\
\hline & MPRGNNTSYI & HLA-B*07:02 & 364 & 373 & 0.00061 \\
\hline \multirow[t]{3}{*}{ WP_001248496.1 } & VTLAQVKVNL & HLA-A*01:01 & 62 & 71 & 0.0516 \\
\hline & MIMTTFPLYY & HLA-A*03:01 & 292 & 301 & 0.00064 \\
\hline & ALMSVSGQFV & HLA-A*02:01 & 1170 & 1179 & 0.00112 \\
\hline \multirow[t]{3}{*}{ WP_000874574.1 } & GISGANGYEK & HLA-A*03:01 & 1214 & 1223 & 0.0193 \\
\hline & TTINLDDSVL & HLA-B*07:02 & 1097 & 1106 & 0.0241 \\
\hline & RYTYKDKFSF & HLA-A*24:02 & 605 & 614 & 0.00031 \\
\hline \multirow[t]{3}{*}{ WP_000902542.1 } & AWCMTQHEGL & HLA-A*24:02 & 768 & 777 & 0.00732 \\
\hline & DHNVLTYFNY & HLA-A*01:01 & 564 & 573 & 0.00841 \\
\hline & YTNLSSQTNY & HLA-A*01:01 & 532 & 541 & $8.79 \mathrm{E}-05$ \\
\hline \multirow[t]{3}{*}{ WP_000915379.1 } & LPYNLNNIEL & HLA-B*07:02 & 135 & 144 & 0.00483 \\
\hline & LPASLFNDPQ & HLA-B*07:02 & 170 & 179 & 0.0212 \\
\hline & YYNHQNIFYY & HLA-A*24:02 & 191 & 200 & 0.00057 \\
\hline \multirow[t]{2}{*}{ WP_000945747.1 } & LSRFVTNMYY & HLA-A*01:01 & 773 & 782 & 0.00221 \\
\hline & TTKGERTFEY & HLA-A*01:01 & 32 & 41 & 0.00358 \\
\hline
\end{tabular}

Table 4. Epitopes for MHC class II predicted by Vaxign for Helicobacter pylori

\begin{tabular}{llllll}
\hline No de acceso & Epitopes & alelo MHC & $\begin{array}{l}\text { aminoacido } \\
\text { inicial }\end{array}$ & $\begin{array}{l}\text { aminoacido } \\
\text { final }\end{array}$ & $\begin{array}{l}\text { Percentil (IEDB } \\
\text { consensus) }\end{array}$ \\
\hline WP_000726314.1 & IQDNYYIDSNIHSQV & HLA-DRB1*04:01 & 496 & 510 & 0.01 \\
& QDNYYIDSNIHSQVQ & HLA-DRB1*04:01 & 497 & 511 & 0.01 \\
\hline WP_000713712.1 & ENIADTLVNFKSRYS & HLA-DRB1*13:01 & 475 & 489 & 0.02 \\
& NIADTLVNFKSRYSE & HLA-DRB1*13:01 476 & 490 & 0.02 \\
\hline WP_001115603 & YAIFQRMYAPGINIT & HLA-DRB1*11:01 & 254 & 268 & 0.13 \\
& FSNKYNIRMDLKLEY & HLA-DRB1*03:01 414 & 428 & 0.01 \\
\hline \multirow{2}{*}{ WP_000709741.1 } & LTPFNQVKSRTIFQL & HLA-DRB1*07:01 & 220 & 234 & 0.01 \\
& NLTPFNQVKSRTIFQ & HLA-DRB1*07:01 & 219 & 233 & 0.01 \\
\hline \multirow{2}{*}{ WP_000822057.1 } & SFNTNYFVIFAKRYY & HLA-DRB1*13:01 & 556 & 570 & 0.01 \\
& FNTNYFVIFAKRYYA & HLA-DRB1*13:01 & 557 & 571 & 0.01 \\
\hline WP_001228436.1 & LINQNALPINYANLS & HLA-DRB1*13:01 & 437 & 451 & 1.52 \\
& VATGLNYRYKHSKYS & HLA-DRB1*08:01 & 602 & 616 & 0.08 \\
\hline WP_001092132.1 & QQYYEYFNNLARMIA & HLA-DRB1*11:01 & 194 & 208 & 0.06 \\
& QYYEYFNNLARMIAL & HLA-DRB1*11:01 & 195 & 209 & 0.06 \\
\hline \multirow{2}{*}{ W10875534.1 } & QLQYRRLYSVYLNYV & HLA-DRB1*01:01 & 591 & 605 & 0.09 \\
\hline & TQLQYRRLYSVYLNY & HLA-DRB1*01:01 & 590 & 604 & 0.13 \\
\hline
\end{tabular}




\begin{tabular}{|c|c|c|c|c|c|}
\hline \multirow{2}{*}{ WP_000911476.1 } & GIKIHIDSNPKFRGL & HLA-DRB1*03:01 & 255 & 269 & 0.11 \\
\hline & EAPGIKIHIDSNPKF & HLA-DRB1*03:01 & 252 & 266 & 0.12 \\
\hline \multirow{2}{*}{ WP_001108275.1 } & VGYWGGLVGQKPWAS & HLA-DRB1*13:01 & 124 & 138 & 0.11 \\
\hline & WGGLVGQKPWASCGL & HLA-DRB1*13:01 & 127 & 141 & 0.11 \\
\hline \multirow{2}{*}{ WP_010875548.1 } & SVNASLQINNIFNMK & HLA-DRB1*13:01 & 749 & 763 & 0.07 \\
\hline & TSTDYFQIFNVMEGG & HLA-DRB1*13:01 & 562 & 576 & 0.45 \\
\hline \multirow{2}{*}{ WP_000479960.1 } & VFIIKLDYTIPKTGI & HLA-DRB1 $* 03: 01$ & 652 & 666 & 0.11 \\
\hline & IIKLDYTIPKTGINL & HLA-DRB1*03:01 & 654 & 668 & 0.28 \\
\hline \multirow{2}{*}{ WP_000753173.1 } & ANFQFLFNMGVRMNL & HLA-DRB1*11:01 & 641 & 655 & 0.61 \\
\hline & NFQFLFNMGVRMNLA & HLA-DRB1*15:01 & 642 & 656 & 0.88 \\
\hline \multirow{2}{*}{ WP_000592437.1 } & SQYLYSLLGAYPTKL & HLA-DRB1*01:01 & 82 & 96 & 0.05 \\
\hline & PTIYNTYYKSAGTTV & HLA-DRB1*07:01 & 485 & 499 & 0.2 \\
\hline \multirow{2}{*}{ WP_000812546.1 } & SQKVRFLAPLSLALS & HLA-DRB1*11:01 & 19 & 33 & 0.15 \\
\hline & QSQKVRFLAPLSLAL & HLA-DRB1*11:01 & 18 & 32 & 0.19 \\
\hline \multirow{2}{*}{ WP_001248496.1 } & EKYFLTSSLSLLSFL & HLA-DRB1 $* 07: 01$ & 4 & 18 & 0.01 \\
\hline & FIDIVTLAQVKVNLL & HLA-DRB1*11:01 & 58 & 72 & 0.34 \\
\hline \multirow{2}{*}{ WP_000874574.1 } & LLNFNGDTTLQNNAN & HLA-DRB1*03:01 & 999 & 1013 & 3.93 \\
\hline & QINTYTQQMSRLAKL & HLA-DRB1*01:01 & 2200 & 2214 & 4.16 \\
\hline \multirow{2}{*}{ WP_000902542.1 } & HRVTGSLQINNIFNM & HLA-DRB1*13:01 & 798 & 812 & 0.07 \\
\hline & TGDPSFIKSLGNNLL & HLA-DRB1 $* 07: 01$ & 217 & 231 & 0.27 \\
\hline \multirow{2}{*}{ WP_000915379.1 } & NLTNLMLNMMAVFDS & HLA-DRB1*13:01 & 259 & 273 & 0.01 \\
\hline & VIYSYRVTNNLYVNL & HLA-DRB1 $* 07: 01$ & 342 & 356 & 0.24 \\
\hline \multirow{2}{*}{ WP_000945747.1 } & FEYNNKMYIDRKELQ & HLA-DRB1 $* 03: 01$ & 39 & 53 & 0.1 \\
\hline & MYIDRKELQQRQSNQ & HLA-DRB1*03:01 & 45 & 59 & 0.24 \\
\hline
\end{tabular}

\section{Conclusions}

In the present study, bioinformatic analysis was made to the complete genome of H. pylori (Strain 26695 with Gen Bank access number of NC_000915). We identified 22 outer membrane proteins using the Vaxign web program. A total of 60 class I epitopes and 40 class II epitopes were predicted. These epitopes could be used in the improvement of active vaccines, for which we consider it important to combine diverse antigens of $\mathrm{H}$. pylori, thus guiding the immune system to defend the host against this bacterium. Accordingly, this study was developed to predict antigenic determinants / epitopes of various outer membrane proteins of $H$. pylori. We propose probable epitopes of B and T cells that can trigger a desired immune response to these proteins. Additionally, they could be used in the design of therapeutic strategies without the use of antibiotics such as passive immunization, in which antibodies are administered to patients to help control infection by this microorganism, which we propose as the first line of action, before use of antibiotics.

\section{Competing Interests Statement}

The authors declare that there are no competing or potential conflicts of interest.

\section{References}

Ayraud, S., Janvier, B., \& Fauchère, J.-L. (2002). Experimental colonization of mice by fresh clinical isolates of Helicobacter pylori is not influenced by the cagA status and the vacA genotype. FEMS Immunology and Medical Microbiology, 34(3), 169-172. Retrieved from http://www.ncbi.nlm.nih.gov/pubmed/12423767

Benson, D. A., Karsch-Mizrachi, I., Lipman, D. J., Ostell, J., \& Wheeler, D. L. (2006). GenBank. Nucleic Acids Research, 34(Database issue), D16-20. https://doi.org/10.1093/nar/gkj157

Chen, F., Mackey, A. J., Stoeckert, C. J., \& Roos, D. S. (2006). OrthoMCL-DB: querying a comprehensive 
multi-species collection of ortholog groups. Nucleic Acids Research, 34(Database issue), D363-8. https://doi.org/10.1093/nar/gkj123

Fallone, C. A., Chiba, N., van Zanten, S. V., Fischbach, L., Gisbert, J. P., Hunt, R. H., ... Marshall, J. K. (2016). The Toronto Consensus for the Treatment of Helicobacter pylori Infection in Adults. Gastroenterology, 151(1), 51-69.e14. https://doi.org/10.1053/j.gastro.2016.04.006

Ford, A. C., \& Axon, A. T. R. (2010). Epidemiology of Helicobacter pylori infection and Public Health Implications. Helicobacter, 15, 1-6. https://doi.org/10.1111/j.1523-5378.2010.00779.x

He, Y., Xiang, Z., \& Mobley, H. L. T. (2010). Vaxign: The first web-based vaccine design program for reverse vaccinology and applications for vaccine development. Journal of Biomedicine and Biotechnology, 2010. https://doi.org/10.1155/2010/297505

Hooi, J. K. Y., Lai, W. Y., Ng, W. K., Suen, M. M. Y., Underwood, F. E., Tanyingoh, D., ... Ng, S. C. (2017). Global Prevalence of Helicobacter pylori Infection: Systematic Review and Meta-Analysis. Gastroenterology, 153(2), 420-429. https://doi.org/10.1053/j.gastro.2017.04.022

Jemilohun, A. C., \& Otegbayo, J. A. (2016). Helicobacter pylori infection: Past, present and future. Pan African Medical Journal. African Field Epidemiology Network. https://doi.org/10.11604/pamj.2016.23.216.8852

Kabir, S. (2011). The Role of Interleukin-17 in the Helicobacter pylori Induced Infection and Immunity. Helicobacter, 16(1), 1-8. https://doi.org/10.1111/j.1523-5378.2010.00812.x

Liao, Y., Deng, J., Zhang, A., Zhou, M., Hu, Y., Chen, H., \& Jin, M. (2009). Immunoproteomic analysis of outer membrane proteins and extracellular proteins of Actinobacillus pleuropneumoniae JL03 serotype 3. BMC Microbiology, 9(1), 172. https://doi.org/10.1186/1471-2180-9-172

Mégraud, F. (2012). The challenge of Helicobacter pylori resistance to antibiotics: the comeback of bismuth-based quadruple therapy. Therapeutic Advances in Gastroenterology, 5(2), 103-109. https://doi.org/10.1177/1756283X11432492

Moss, S. F., Moise, L., Lee, D. S., Kim, W., Zhang, S., Lee, J., ... De Groot, A. S. (2011). HelicoVax: epitope-based therapeutic Helicobacter pylori vaccination in a mouse model. Vaccine, 29(11), 2085-2091. https://doi.org/10.1016/j.vaccine.2010.12.130

Ni, X. D., Wang, N., Liu, Y. J., \& Lu, C. P. (2010). Immunoproteomics of extracellular proteins of the Aeromonas hydrophila China vaccine strain J-1 reveal a highly immunoreactive outer membrane protein. FEMS Immunology and Medical Microbiology, 58(3), 363-373. https://doi.org/10.1111/j.1574-695X.2009.00646.x

Pilotto, A., \& Franceschi, M. (2014). Helicobacter pylori infection in older people. World Journal of Gastroenterology, 20(21), 6364-6373. https://doi.org/10.3748/wjg.v20.i21.6364

Pruitt, K. D., Tatusova, T., \& Maglott, D. R. (2005). NCBI Reference Sequence (RefSeq): a curated non-redundant sequence database of genomes, transcripts and proteins. Nucleic Acids Research, 33(Database issue), D501-4. https://doi.org/10.1093/nar/gki025

Refaeli, R., Chodick, G., Haj, S., Goren, S., Shalev, V., \& Muhsen, K. (2018). Relationships of H. pylori infection and its related gastroduodenal morbidity with metabolic syndrome: a large cross-sectional study. Scientific Reports, 8(1), 4088. https://doi.org/10.1038/s41598-018-22198-9

Sachdeva, G., Kumar, K., Jain, P., \& Ramachandran, S. (2005). SPAAN: a software program for prediction of adhesins and adhesin-like proteins using neural networks. Bioinformatics, 21(4), 483-491. https://doi.org/10.1093/bioinformatics/bti028

Spohn, G., \& Scarlato, V. (2001). Motility, Chemotaxis, and Flagella. Helicobacter pylori: Physiology and Genetics. ASM Press. Retrieved from http://www.ncbi.nlm.nih.gov/pubmed/21290725

Xiang, Z., \& He, Y. (2009). Vaxign: a web-based vaccine target design program for reverse vaccinology. Procedia in Vaccinology, 1(1), 23-29. https://doi.org/10.1016/j.provac.2009.07.005

Xiang, Z., \& He, Y. (2013). Genome-wide prediction of vaccine targets for human herpes simplex viruses using $\begin{array}{llllllll}\text { Vaxign reverse vaccinology. BMC Bioinformatics, } 14 & \text { Suppl 4(4), } & \text { S2. }\end{array}$ https://doi.org/10.1186/1471-2105-14-S4-S2

Yu, N. Y., Wagner, J. R., Laird, M. R., Melli, G., Rey, S., Lo, R., ... Brinkman, F. S. L. (2010). PSORTb 3.0: improved protein subcellular localization prediction with refined localization subcategories and predictive capabilities for all prokaryotes. Bioinformatics (Oxford, England), 26(13), 1608-1615. 
https://doi.org/10.1093/bioinformatics/btq249

\section{Appendix}

Table 1s. Comparison of four software for prediction of epitopes MHC I and MHC II classes of Helicobacter pylori

\begin{tabular}{|c|c|c|c|c|}
\hline \multirow[t]{2}{*}{ Accession \# } & \multirow{2}{*}{ sofware } & \multicolumn{2}{|c|}{ predicion petidos } & \multirow[t]{2}{*}{ metodo de prediciòn } \\
\hline & & MHC I & MHC II & \\
\hline \multirow{4}{*}{ WP_000726314.1 } & Vaxign & 90 & 86 & PSSM \\
\hline & epijen v1.0 & 40 & & AGMP \\
\hline & CTLPred & 28 & & ANNs y SVMs \\
\hline & imtech & & 3 & MCTT y ANNs \\
\hline \multirow{4}{*}{ WP_000713712.1 } & Vaxign & 92 & 131 & PSSM \\
\hline & epijen v1.0 & 42 & & AGMP \\
\hline & CTLPred & 29 & & ANNs y SVMs \\
\hline & imtech & & 3 & MCTT y ANNs \\
\hline \multirow{4}{*}{ WP_001115603 } & Vaxign & 94 & 112 & PSSM \\
\hline & epijen v1.0 & 28 & & AGMP \\
\hline & CTLPred & 29 & & ANNs y SVMs \\
\hline & imtech & & 51 & MCTT y ANNs \\
\hline \multirow{4}{*}{ WP_000709741.1 } & Vaxign & 66 & 48 & PSSM \\
\hline & epijen v1.0 & 18 & & AGMP \\
\hline & CTLPred & 17 & & ANNs y SVMs \\
\hline & imtech & & 34 & MCTT y ANNs \\
\hline \multirow{4}{*}{ WP_000822057.1 } & Vaxign & 186 & 154 & PSSM \\
\hline & epijen v1.0 & 51 & & AGMP \\
\hline & CTLPred & 36 & & ANNs y SVMs \\
\hline & imtech & & 4 & MCTT y ANNs \\
\hline \multirow{4}{*}{ WP_001228436.1 } & Vaxign & 151 & 134 & PSSM \\
\hline & epijen v1.0 & 28 & & AGMP \\
\hline & CTLPred & 32 & & ANNs y SVMs \\
\hline & imtech & & 68 & MCTT y ANNs \\
\hline \multirow{4}{*}{ WP_001092132 } & Vaxign & 114 & 99 & PSSM \\
\hline & epijen v1.0 & 28 & & AGMP \\
\hline & CTLPred & 23 & & ANNs y SVMs \\
\hline & imtech & & 51 & MCTT y ANNs \\
\hline \multirow{4}{*}{ WP_010875534.1 } & Vaxign & 122 & 109 & PSSM \\
\hline & epijen v1.0 & 27 & & AGMP \\
\hline & CTLPred & 32 & & ANNs y SVMs \\
\hline & imtech & & 61 & MCTT y ANNs \\
\hline
\end{tabular}




\begin{tabular}{|c|c|c|c|c|}
\hline \multirow{4}{*}{ WP_000911476.1 } & Vaxign & 112 & 110 & PSSM \\
\hline & epijen v1.0 & 26 & & AGMP \\
\hline & CTLPred & 26 & & ANNs y SVMs \\
\hline & imtech & & 48 & MCTT y ANNs \\
\hline \multirow{4}{*}{ WP_001108275.1 } & Vaxign & 118 & 96 & PSSM \\
\hline & epijen v1.0 & 27 & & AGMP \\
\hline & CTLPred & 21 & & ANNs y SVMs \\
\hline & imtech & & 52 & MCTT y ANNs \\
\hline \multirow{4}{*}{ WP_010875548.1 } & Vaxign & 174 & 155 & PSSM \\
\hline & epijen v1.0 & 27 & & AGMP \\
\hline & CTLPred & 36 & & ANNs y SVMs \\
\hline & imtech & & 81 & MCTT y ANNs \\
\hline \multirow{4}{*}{ WP_000479960.1 } & Vaxign & 184 & 132 & PSSM \\
\hline & epijen v1.0 & 27 & & AGMP \\
\hline & CTLPred & 30 & & ANNs y SVMs \\
\hline & imtech & & 82 & MCTT y ANNs \\
\hline \multirow{4}{*}{ WP_000753173.1 } & Vaxign & 142 & 126 & PSSM \\
\hline & epijen v1.0 & 27 & & AGMP \\
\hline & CTLPred & 16 & & ANNs y SVMs \\
\hline & imtech & & 73 & MCTT y ANNs \\
\hline \multirow{4}{*}{ WP_000592437.1 } & Vaxign & 102 & 88 & PSSM \\
\hline & epijen v1.0 & 27 & & AGMP \\
\hline & CTLPred & 27 & & ANNs y SVMs \\
\hline & imtech & 55 & & MCTT y ANNs \\
\hline \multirow{4}{*}{ WP_000812546.1 } & Vaxign & 112 & 113 & PSSM \\
\hline & epijen v1.0 & 27 & & AGMP \\
\hline & CTLPred & 26 & & ANNs y SVMs \\
\hline & imtech & & 57 & MCTT y ANNs \\
\hline \multirow{4}{*}{ WP_001248496.1 } & Vaxign & 127 & 110 & PSSM \\
\hline & epijen v1.0 & 29 & & AGMP \\
\hline & CTLPred & 24 & & ANNs y SVMs \\
\hline & imtech & & 55 & MCTT y ANNs \\
\hline \multirow{4}{*}{ WP_000874574.1 } & Vaxign & 514 & 506 & PSSM \\
\hline & epijen v1.0 & 130 & & AGMP \\
\hline & CTLPred & 96 & & ANNs y SVMs \\
\hline & imtech & & 249 & MCTT y ANNs \\
\hline \multirow{4}{*}{ WP_000902542.1 } & Vaxign & 189 & 143 & PSSM \\
\hline & epijen v1.0 & 46 & & AGMP \\
\hline & CTLPred & 36 & & ANNs y SVMs \\
\hline & imtech & & 89 & MCTT y ANNs \\
\hline WP_000915379.1 & Vaxign & 167 & 122 & PSSM \\
\hline
\end{tabular}




\begin{tabular}{lllll}
\hline & epijen v1.0 & 59 & AGMP \\
\cline { 2 - 4 } CTLPred & 46 & 112 & ANNs y SVMs \\
\cline { 2 - 4 } imtech & & 148 & MCTT y ANNs \\
\hline VP_000945747.1 & Vaxign & 193 & AGMP \\
\cline { 2 - 4 } & epijen v1.0 & 47 & ANNs y SVMs \\
\cline { 2 - 4 } & CTLPred & 36 & MCTT y ANNs \\
\hline
\end{tabular}

\section{Copyrights}

Copyright for this article is retained by the author(s), with first publication rights granted to the journal.

This is an open-access article distributed under the terms and conditions of the Creative Commons Attribution license (http://creativecommons.org/licenses/by/4.0/). 